\title{
The Study of Size Distribution and Spatial Distribution of Urban Systems in Guangdong, China
}

\author{
Jianmei Yang, Dong Zhuang, and Minyi Kuang \\ School of Business Administration, \\ Institute of Emerging Industrialization Development, \\ South China University of Technology, P.R. China, 510640
}

\begin{abstract}
There are three types of urban size distribution: primate city distribution, rank-size distribution and the medium distribution between them. In order to avoid the defect of data, the paper uses both the population and built-up district area data of above county level cities in Guangdong Province in 2002 and 2004. The results prove that urban size of the urban system in the province follows rank-size distribution. In analyzing the value and the change of Zipf exponents, we point out the characteristics of the size distribution of urban system in the province. The paper also conducts spatial distribution analysis for urban system in Guangdong Province. Power law distribution is also observed, and value of the exponent belongs to the same range of value with those in the size distribution analysis. The differences of focal point between fractal dimension (the inverse of Zipf exponent) and power law exponent (prevailing in complex network analysis) are finally discussed.
\end{abstract}

Keywords: Power law distribution, urban size distribution, urban spatial distribution, complex networks.

\section{Introduction}

Guangdong Province is situated in the south of China P.R.C, adjacent to Hong Kong and Macao, with a total area of 1,797.57 hectares. According to the National Census report of the year 2005, there are 91.85 million permanent residents in Guangdong Province at 0:00, November 1st of the year 2005. Compared with the 86.42 million of that in the year 2000 in the fifth National Census, the population is increased by 5.43 million, amount for $6.28 \%$, increasing 109 million per year and the annual grow rate is $1.23 \%$. Until the end of 2004 , there are totally 21 prefecture-level cities, 23 county-level cities, 41 counties and 3 autonomous counties in Guangdong Province.

Since 1995, Guangdong accelerated its steps in urbanization. At the end of 2005, the urban population of Guangdong Province reached 55.73 million, amount for $60.68 \%$ of the total population. However, there is great difference among different region in the province. The Pearl River Delta has high urbanization level with prominent urban cluster appearance, while for other regions 
in the province, especially the northern mountain area, gaps are large compared to the former. The new development of city systems in Guangdong needs to be analyzed scientifically by using international common practice.

\section{Urban Size Distribution Model and Spatial Distribution Model}

Zipf distribution. We know that the larger the city, the smaller the amount, while small cities are huge in quantity. In 1949, G. K. Zipf [1] suggested the ranksize rule, which measures the city size distribution in a city system in terms of the relationship between the size and the rank of the city, and argued the power law distribution between the two variables

$$
P_{R}=P_{1} / R
$$

In formula (1), $P_{R}$ denotes the population of the city, $P_{1}$ the population of the largest city theoretically, and $R$ the rank of city with $P_{R}$. More generally, the Zipf's law can be denoted as

$$
P_{i}=P_{1} \cdot R_{i}^{-q}
$$

where $P_{i}$ denotes the population of city $i, P_{1}$ the population of the largest city, $R_{i}$ the rank of city $i$, and $q$ is a constant.

Pareto distribution. If we use a population yardstick $r$ to depict the size of cities, and let $P(r)$ denote the probability of $S>r$ (i.e., a city having a population above $r), P(s)$ denote the probability of city size distribution, then we get

$$
P_{r} \propto \int_{r}^{\infty} P(s) d s .
$$

If for any $\lambda>0, P(\lambda r) \propto \lambda^{-a} P(r)$, where $\lambda$ denotes the proportion of scale, the scaling exponent, then we get the Pareto distribution of city size [2]

$$
P_{r} \propto r^{-a},
$$

where $P(r)$ is the cumulative percentage of cities above threshold $r$.

If the total quantity of cities is $N_{0}$, let $P(r)=N(r) / N_{0}$, then (4) can be rewritten as

$$
N_{r} \propto C \cdot r^{-D}
$$

where $N(r)$ is the cumulative quantity of cities, and $C$ is a constant [2].

Compared with Hausdorff dimension formula, we know $D$ (equals $\alpha$ ) is the fractal dimension [3], 4]. Expression (5]) is equivalent to the general form the Zipf formula

$$
P(K)=P_{1} K^{-q} .
$$

In formula (6), $K$ denotes the rank of cities, $q$ the Zipf exponent. Theoretically, $q=1 / \alpha$ has the fractal dimensional properties, therefore it is called the Zipf dimension. 
Spatial distribution model. The spatial correlation function can be expressed in the formula

$$
\begin{array}{r}
C(r)=\frac{1}{N^{2}} \sum_{i}^{N} \sum_{i}^{N} H\left(r-d_{i j}\right), \text { where } i \neq j \\
\text { and }\left(r-d_{i j}\right)=\left\{\begin{array}{l}
1, d_{i j} \leq r \\
0, d_{i j}>r
\end{array}\right.
\end{array}
$$

Here $r$ is an yardstick, $N$ is the total amount of cities, $d_{i j}$ represents the Euclidian distance (or, Crow Distance) between city $i$ and $j$, and $H$ is Heaviside function [5]. To facilitate calculation, formula (7) can be modified to

$$
C(r)=\sum_{i}^{N} \sum_{i}^{N} H\left(r-d_{i j}\right) \text {, where } i \neq j .
$$

If there is scale invariance in the spatial distribution of urban system, then

$$
\begin{gathered}
C(\lambda r) \propto \lambda^{D} C(r), \\
C(r) \propto r^{D},
\end{gathered}
$$

where $D$ is the fractal dimension of the urban spatial distribution.

Formula (9) suggests that in an urban system, the frequency for distance between cities to be below $r$ follows power law distribution. $D$ represents the extent of differences in the distance between cities, or the extent of balance in the urban system spatial distribution.

Here follows the analysis of size distribution and spatial distribution of Guangdong urban system.

\section{Size Distribution of Urban System in Guangdong Province and the Search for the Exponent}

\subsection{Calculation Method and Data Source}

Calculation method. There are two ways to measure the urban size. One is to measure the size of urban population. In China, the urbanization level is generally measured by the proportion of non-agricultural population in the total population in the city, so the non-agricultural population is generally used to measure the urban size. Another method is to use the built-up district area as the measure.

The traditional rank-size distribution always uses the former method. Empirical studies show that research based on the more stable non-agricultural population data can better reflect urban size and its growth speed. However, the defects are also obvious. In the case of cities which have more floating population 
than that of the permanent residents, such method can hardly reflect actual situation. For example, the floating populations in Shenzhen and Dongguan take large proportion of the total population. In statistical data from the sampled National population survey of 2005, for Shenzhen in 2004, the non-agricultural population is 1.6477 million, with registered population 1.6513 million, while the actual population (including floating population) in the city reached 8.2775 million. Therefore, merely using non-agricultural population may have high inaccuracy. The built-up district area appears stable in the development of a city, and it can better reflect the scale of the city since its statistics are less interfered by other factors. For this reason, some foreign scholars used the built-up district area in the rank-size analysis to study the development of urban cluster. When applying the rank-size rule to analyze the development of built-up district in Berlin, Schweitze etal pointed out that urban clusters are composed of many built-up districts with different area, and that the area and the rank of the city follows rank-size rule 6].

Considering the applying contexts and advantage of both methods, based on the current population situation in Guangdong Province, we use both methods to analyze the urban size distribution in the province, and the results are then compared.

Data source. The study focuses on the above county-level cities in Guangdong Province in 2002 and 2004. Data are collected from the Guangdong Statistical Yearbook. The population data concern the registered non-agriculture population. Because the built-up district areas of below prefecture-level cities are not recorded, we only use the data of above prefecture-level cities in the analysis, not including the counties and towns administrated by them.

\subsection{Characteristics of the Distribution and the Exponents}

According to the data collected, cities are ranked by their urban populations and built-up district areas, respectively. The results are log-log plotted in Fig. 1]and2, From the figures, we see that all the distributions can be fitted by a straight line. Using formula

$$
\ln P(K) \propto-q \ln K,
$$

we can get the Zipf dimension $q$, see Tab. 1 .

Table 1. Main indices of urban size distribution in Guangdong Province

\begin{tabular}{c|c|c|c|c|c}
\hline Measure & Year & Quantity of cities & $q$ & $R$ & $D$ \\
\hline \multirow{2}{*}{ Population } & 2002 & 92 & 0.825 & 0.954 & 1.212 \\
\cline { 2 - 6 } & 2004 & 93 & 0.923 & 0.957 & 1.083 \\
\hline \multirow{2}{*}{ Built-up district area } & 2002 & 21 & 0.773 & 0.969 & 1.294 \\
\cline { 2 - 6 } & 2004 & 21 & 0.764 & 0.976 & 1.309 \\
\hline
\end{tabular}




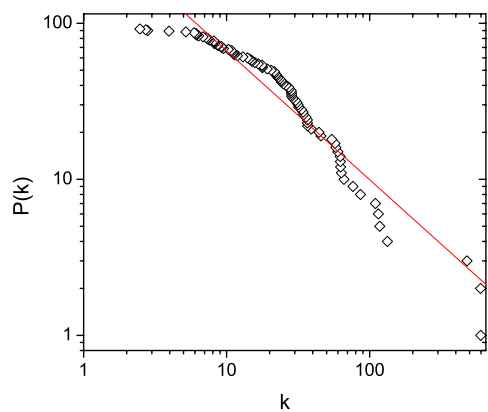

(a)

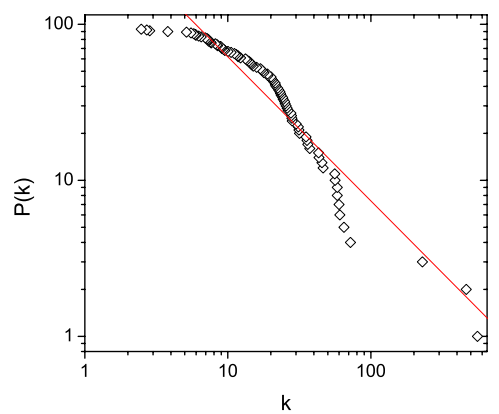

(b)

Fig. 1. Log-log rank-size distribution of urban population in Guangdong Province (k: rank of the cities; $\mathrm{P}(\mathrm{k})$ : urban populations of the cities). (a) in the year of 2002; (b) in the year of 2004 .

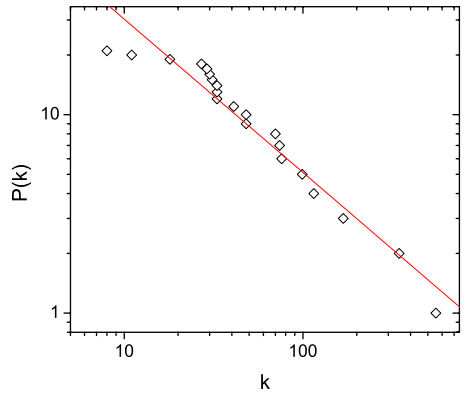

(a)

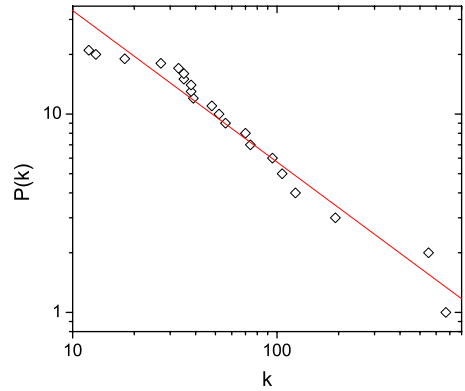

(b)

Fig. 2. Log-log rank-size distribution of built-up district areas in Guangdong Province (k: rank of the cities; $\mathrm{P}(\mathrm{k})$ : urban population of the cities). (a) in the year of 2002 ; (b) in the year of 2004 .

From the analysis above, it is proved that the urban sizes of Guangdong Province follow size-rank distribution in terms of both urban population and built-up district area, and Zipf exponents are all below 1. From 2002 to 2004, the population index increased, while the area index decreased.

\section{Spatial Distribution of Urban System in Guangdong Province and the Search for the Exponent}

The spatial distribution of major cities takes the above prefecture-level cities in Guangdong Province. In consideration of the particularity of the administrative 
division in Foshan, two districts administrated by it (i.e., the Sanshui district and the Shunde district) are treated as cites. Then there are totally 23 cities.

In studying the spatial distances between cities, the Googly-Earth Satellite software is used to measure the accurate distances between city $i$ and $j$, denoted by $d_{i j}$, which are filled into a $23 \times 23$ symmetric matrix. By assigning $r$ with the multiple of 30 (i. e., $r=30,60,90, \cdots, 720$ kilometers), we may obtain a series of corresponding $C(r)$ following formula 8, as listed in Tab. 2, The data are plotted and fitted on a log-log scale (see Fig. 3).

Table 2. Distance $r$ and correlation function $C(r)$

\begin{tabular}{c|c|c|c|c|c|c|c|c}
\hline$r$ & 30 & 60 & 90 & 120 & 150 & 180 & 210 & 240 \\
\hline$C(r)$ & 10 & 40 & 94 & 132 & 178 & 222 & 258 & 286 \\
\hline$r$ & 270 & 300 & 330 & 360 & 390 & 420 & 450 & 480 \\
\hline$C(r)$ & 310 & 342 & 362 & 418 & 442 & 452 & 464 & 474 \\
\hline$r$ & 510 & 540 & 570 & 600 & 630 & 660 & 690 & 720 \\
\hline$C(r)$ & 478 & 488 & 490 & 492 & 498 & 498 & 504 & 506 \\
\hline
\end{tabular}

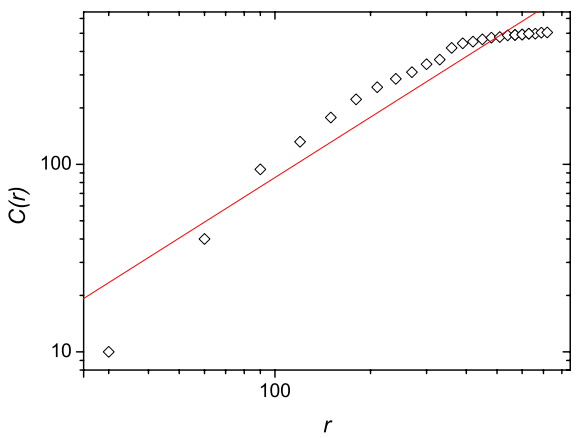

Fig. 3. Log-log plot of correlation function $C(r)$ vs. distance $r$

The regression equation is $\ln C(r)=-0.21+1.07 \ln r$, with correlation coefficient 0.91 and power law exponent (i.e. the fractal dimension) 1.07.

\section{The Implication for the Power Law Distribution of Guangdong Urban Systems}

According to existing research, all the early observed urban rank-size distributions have $q=1$, and urban size distributions in many developed countries have dimensions approaching 1. While for developing countries, the dimensions are far from 1 . That's why the science of urban planning takes 1 as the natural or 
ideal Zipf exponent. Therefore, whether $q$ equals 1 should be used to assess the rank-size distribution.

From Tab. 1, we obtained the $q, R$ and $D$ values for the size distribution of urban system in Guangdong Province. In terms of the science of urban planning, the distribution of population is more ideal than that of the built-up district area. The urban population distribution is getting more reasonable, while that of the built-up district area has an opposite tendency.

In terms of the complex network studies in recent years [7], cumulative power law exponent between 1 and 2 results in the coexistence of small amount of largesized nodes and large quantities of small-sized nodes. When the value is below 1 , there are quite a few large-sized nodes. In the case of study for Guangdong urban systems, the distribution follows the former. That is, a few large cities coexist with a large amount of small cities.

The fractal dimension of Guangdong urban system spatial distribution $D$ is 1.07, even smaller than that of the population. It suggest that the variance of urban spatial distribution is greater than those of the size distributions measured by population and built-up district area.

From 2002 to 2004, the power law exponent of population distribution is increasing with that of the built-up district area distribution decreasing, suggesting the trend for the variance of population size to decrease and that of the built-up district area size to increase. The distribution exponent of population to approach 1 suggests the evolution trends for the population size distribution to getting closer to those in the developed countries and regions.

\section{Conclusion and Discussion}

To summarize, both the urban size and the spatial in Guangdong Province follow power law distribution, with the accumulative power law exponent between 1 and 2. It suggests the self-organized critical state [8] of urban distribution in the Guangdong urban systems, with prominent fractal characteristics, and with the coexistence of a handful of large cities with large amount of small cities.

Because floating populations are not included in the study (without access to obtain the data), there must be errors in the conclusion. Therefore, size distribution considering built-up district area should also be combined in the analysis.

We previously pointed out that the Zipf exponent $q$ is the inverse of Pareto exponent (i.e. D), and Pareto distribution has the same implications with cumulative degree distribution in complex network. In complex network studies, networks with cumulative distribution exponents between 1 and 2 are concerned most, because their robust yet fragile feature can be significant in practice. However, in the science of urban planning, whether the Pareto exponent (i.e. $D$ ) equals 1 is concerned most(compared with urban systems having $D$ between 1 and 2, they have more large size cities). The practical significance and theoretical context of this difference, and why urban systems with $D=1$ are the most reasonable need more further research to be done. 


\section{References}

1. Zipf, G. K.: Human Behavior and the Principle of least effort, Addison-Wesley, Reading, MA (1949)

2. Shiode, N., Batty, M.: Power Law Distributions in Real and Virtual Worlds, INET'2000 (2000)

3. Mandelbrot, B.B.: The Fractal Geometry of Nature. W. H. Freeman and Company, New York (1983)

4. Batty, M., Longley, P.A., Fotheringham, A.S.: Urban growth and form: scaling, fractal geometry, and diffusion-limited aggregation. Environment and Planning A, 21 (1989) 1447-1472

5. Liu, J.S., Chen, Y.G.: A study on Fractal Dimension of spatial Structure of Trasport Networks and the Methods of Their Determination. Acta Geographica sinica, Vol. 54, 5 (1999)

6. Schweitzer, F., Steinbrink, J.: Estimation of megacity growth: simple rules versus complex phenomena. Applied Geography, (1998) 18(1): 69-81

7. Barabási, A. L., Albert, R.: Emergence of scaling in random networks. Science Vol. 286 (1999) 509

8. Portugali, J.: Self-Organization and the City. Springer-Verlag, Berlin (2000) 